\title{
Removal of Matrix Interferences by Nano-MgO and Co-Adsorbents for Accurate Multi-Pesticide Residue Analysis in the Chinese Medicinal Herb, Paeoniae Radix Alba
}

\author{
Chunyu Wang, ${ }^{1}$ Xinquan Wang $\mathbb{D}^{2,3,4}$ Jiao Wang, ${ }^{2,3,4}$ Shanshan $\mathrm{Di}^{2,3,4}$ Zhiwei Wang, ${ }^{2,3,4}$ \\ Hao Xu, ${ }^{2,3,4}$ Huiyu Zhao, ${ }^{2,3,4}$ Changshan Zhao ${ }^{1 D}$, ${ }^{1}$ and Peipei Qi $\mathbb{D}^{2,3,4}$ \\ ${ }^{1}$ College of Agriculture, Northeast Agricultural University, Harbin 150030, China \\ ${ }^{2}$ State Key Laboratory for Managing Biotic and Chemical Threats to the Quality and Safety of Agro-products, \\ Institute of Quality and Standard of Agro-products, Zhejiang Academy of Agricultural Sciences, Hangzhou 310021, China \\ ${ }^{3}$ Agricultural Ministry Key Laboratory for Pesticide Residue Detection, Hangzhou 310021, China \\ ${ }^{4}$ Key Laboratory of Detection for Pesticide Residue and Control of Zhejiang, Hangzhou 310021, China
}

Correspondence should be addressed to Changshan Zhao; csz-hlj@sohu.com and Peipei Qi; qipeipei@zaas.ac.cn

Received 5 November 2020; Revised 22 December 2020; Accepted 31 December 2020; Published 5 February 2021

Academic Editor: Cecilia Cagliero

Copyright $\odot 2021$ Chunyu Wang et al. This is an open access article distributed under the Creative Commons Attribution License, which permits unrestricted use, distribution, and reproduction in any medium, provided the original work is properly cited.

A simple, accurate, and high-throughput analytical method was developed to detect 123 pesticide residues in Chinese medicinal herb Paeoniae Radix Alba (PRA) by introducing nano-MgO as a highly efficient purification material based on quick, easy, cheap, effective, rugged, and safe (QuEChERS) design concept. Various PRA samples were extracted using $8 \mathrm{~mL} 0.5 \%$ acetic acidacetonitrile solution and purified by a dispersive solid-phase extraction method with $30 \mathrm{mg}$ nano-MgO, $40 \mathrm{mg}$ primary secondary amine (PSA), and $40 \mathrm{mg}$ octadecylsilane (C18) as the cleanup adsorbents, followed by liquid chromatography-tandem mass spectrometry (LC-MS/MS). $70.7 \%$ of pesticides showed a weak matrix effect after the purification process, indicating that this method can give the precise quantitative analysis of trace pesticides residue. The method was systematically validated under optimal conditions in five different kinds of PRA samples; good linearity was observed in the concentration range of $0.5-250 \mu \mathrm{g} / \mathrm{L}$ or $1-250 \mu \mathrm{g} / \mathrm{L}$. Pesticide recovery in each sample spiked at concentrations of 20,50 , and $200 \mu \mathrm{g} / \mathrm{kg}$ ranged from $98.0 \%$ to $111 \%$ and the mean relative standard deviation ranged from $2.72 \%$ to $5.70 \%$. Furthermore, the method comparison with the traditional QuEChERS method suggested the feasibility, advantages, and potential application prospect of the present method for the multipesticide residue analysis in various PRA samples.

\section{Introduction}

The medicinal herb is playing an increasingly important role in our daily life, as a therapeutic drug, a dietary supplement, or a natural cosmetics additive $[1,2]$. Traditional Chinese medicinal (TCM) herbs have been used to cure diseases for more than 2000 years in China [3], and 8980 medicinal herbs were recorded in the classic book Chinese Materia Medica. Paeoniae Radix Alba (PRA) is the dried root of Paeonia lactiflora Pall; the family is Ranunculaceae. As one of the most commonly used TCM, PRA has displayed multiple pharmacological functions, such as easing pain, nourishing blood, and treating depressive disorder [4-6]. It has also been used in cosmetics as an anti-aging ingredient in recent decades. Hence, with the extended application areas and the increasing demands, the artificial cultivation area of PRA is gradually expanding. During its cultivation, the use of pesticides is inevitable to avoid the infestation of disease, insect, and grass and consequently increase productivity [7]. Until now, China is the main producer and exporter of PRA. However, there is no pesticide registered on PRA in China; the abuse of pesticides has become a universal phenomenon, leading to serious pesticide residues in PRA, such as organophosphorus pesticides (e.g., dimethoate, chlorpyrifos, 
disulfoton, terbufos, and malathion) [8, 9], organochlorine pesticides [10, 11], and carbamates pesticides (e.g., carbendazim) [2]. Therefore, a reliable, simple, and highthroughput detection method is urgently needed to monitor the pesticide residue in PRA and ensure its product safety.

PRA contains many different types of ingredients, such as paeoniflorin, benzoic acid, volatile oils, and fatty oils, which makes it owning multiple functions [4]. However, the extraction of target analytes is usually accompanied by the co-extraction of these complex components, making an accurate analysis of trace pesticide residues difficult. Currently, traditional solid-phase extraction (SPE) [11], hollow fiber-based liquid-phase microextraction (HF-LPME) [12], and dispersive solid-phase extraction (dSPE) [13] have been used for pesticide residue analysis in PRA. Relatively, SPE and HF-LPME are cumbersome to operate. dSPE is more effective in purifying the sample. Solvent extraction coupled with dSPE purification was a typical quick, easy, cheap, effective, rugged, and safe (QuEChERS) method, which has become a widely accepted design concept for pesticide residue analysis in various matrices [14-17]. Currently, several studies focused on the analysis of pesticide residue in PRA based on the QuEChERS method. However, the complexity of the PRA matrix made the low pesticide coverage of these developed methods and the poor applicability for different kinds of PRA. Therefore, new efficient purification material was urgently needed to remove the matrix interferences and improve the method accuracy, efficiency, and applicability.

Nano-MgO has been reported as an excellent adsorbent to adsorb chemical pollutants such as humic acid [18] and phosphate [19] from water samples, likely due to its amphiphilic character. According to the Lewis acid-base theory, $\mathrm{Mg}^{2+}$ at Lewis acid sites can interact with alkaline substances, while $\mathrm{O}^{2-}$ at Lewis basic sites can adsorb acidic substances [20, 21]. This characteristic lets us consider the feasibility of nano-MgO in the removal of the acid active compounds in the PRA matrix. Meanwhile, as a nanooxidation material, its small particle size and large specific surface area can help to enhance its adsorption performance. Besides, owing to the diversity of the active compounds in the PRA matrix, the coadsorbents were beneficial to improve the purification effect and commercial C18 and PSA can eliminate the interference of oils and weak polar compounds, respectively.

The present work was aimed at establishing a rapid method for the detection of 123 pesticide residues in various PRA samples based on the selection of purification materials according to the QuEChERS design concept, followed by liquid chromatography-tandem mass spectrometry (LCMS/MS) analysis. The 123 common pesticides that may be used in PRA cultivation were selected as target analytes, including organophosphorus, carbamate, nicotine, triazole, and amides. Both the extraction and purification steps were systematically optimized, and nano-MgO was innovatively used as a dSPE adsorbent to reply to the complexity of the PRA sample, significantly reducing the matrix effect. Moreover, the performance of the established method was validated and compared with the traditional QuEChERS method to verify its feasibility in pesticide residue analysis in PRA samples.

\section{Experimental}

2.1. Materials. Five different PRA samples were purchased from diverse main producing areas according to its planting situation, including Zhejiang, Anhui, Shandong, Henan, and Hebei provinces in China. The samples were named PRA-ZJ, PRA-AH, PRA-SD, PRA-HN, and PRA-HB, respectively. The samples were pulverized by a grinder and sieved through a 100-mesh sieve, and PRA powder was stored in a desiccator at room temperature until measured.

2.2. Chemicals and Regents. Nano-MgO was obtained from Nanjing XFNANO Materials Tech Co., Ltd. (Nanjing, China). High-performance liquid chromatography (HPLC) grade acetonitrile $(\mathrm{ACN})$, methanol, and isopropanol were purchased from Merck (Darmstadt, Germany). Acetic acid (HAc) was of analytical grade and was purchased from YONGHUA Chemical Co., Ltd. (Jiangsu, China). Primary secondary amine (PSA), octadecylsilane (C18), anhydrous magnesium sulfate $\left(\mathrm{MgSO}_{4}\right)$, and sodium chloride $(\mathrm{NaCl})$ were obtained from Bonna-Agela Technologies Co. Ltd. (Tianjin, China). HPLC grade ammonium formate was purchased from Tedia (Fairfield, USA). Ultrapure water was used throughout the experiment. 123 pesticides were purchased from the Ministry of Agriculture, Agro-Environmental Protection Institute (Tianjin, China), or Shanghai Pesticide Research Institute (Shanghai, China). All pesticides were formulated as a mixed standard of $5 \mathrm{mg} / \mathrm{L}$ and stored in a refrigerator at $4^{\circ} \mathrm{C}$.

2.3. Sample Preparation. The PRA powder ( $2 \mathrm{~g})$ was weighed into $50 \mathrm{~mL}$ centrifuge tubes, followed by the addition of $10 \mathrm{~mL}$ of water and $8 \mathrm{~mL}$ of a $0.5 \%$ acetic acid-acetonitrile solution for the extraction process. The mixture was vortexed for $1 \mathrm{~min}$ to ensure adequate contact between the extraction solvent and the sample. Then, $6 \mathrm{~g}$ of anhydrous $\mathrm{MgSO}_{4}$ and $1.5 \mathrm{~g}$ of $\mathrm{NaCl}$ were added to the tube and vortexed for $1 \mathrm{~min}$. The resulting mixture was centrifuged at $7000 \mathrm{~g}$ for $5 \mathrm{~min}$ using a Thermo Scientific Biofuge Primo $R$ centrifuge (Germany).

The supernatant acetonitrile $(1 \mathrm{~mL})$ in the extraction solution was transferred into $2 \mathrm{~mL}$ centrifuge tubes containing $30 \mathrm{mg}$ of nano-MgO, $40 \mathrm{mg}$ of PSA, $40 \mathrm{mg}$ of $\mathrm{C} 18$, and $150 \mathrm{mg}$ of anhydrous $\mathrm{MgSO}_{4}$. After being vortexed for $1 \mathrm{~min}$, the resulting mixture was centrifuged at $7000 \mathrm{~g}$ for $5 \mathrm{~min}$. Later, $600 \mu \mathrm{L}$ of supernatant was mixed with $150 \mu \mathrm{L}$ of water. The solution was filtered through $0.22 \mu \mathrm{m}$ filters before the LC-MS/MS analysis. The scheme of the established pretreatment method procedure is shown in Figure 1.

During the method optimization, PRA-ZJ was used as a typical sample and it was spiked with 123 pesticides at $0.2 \mathrm{mg} / \mathrm{kg}$. First, the types and volume of extraction solvents were optimized for sample solution preparation. The extraction solvent was directly analyzed by LC-MS/MS without purification. Subsequently, the amounts of dSPE adsorbents, 


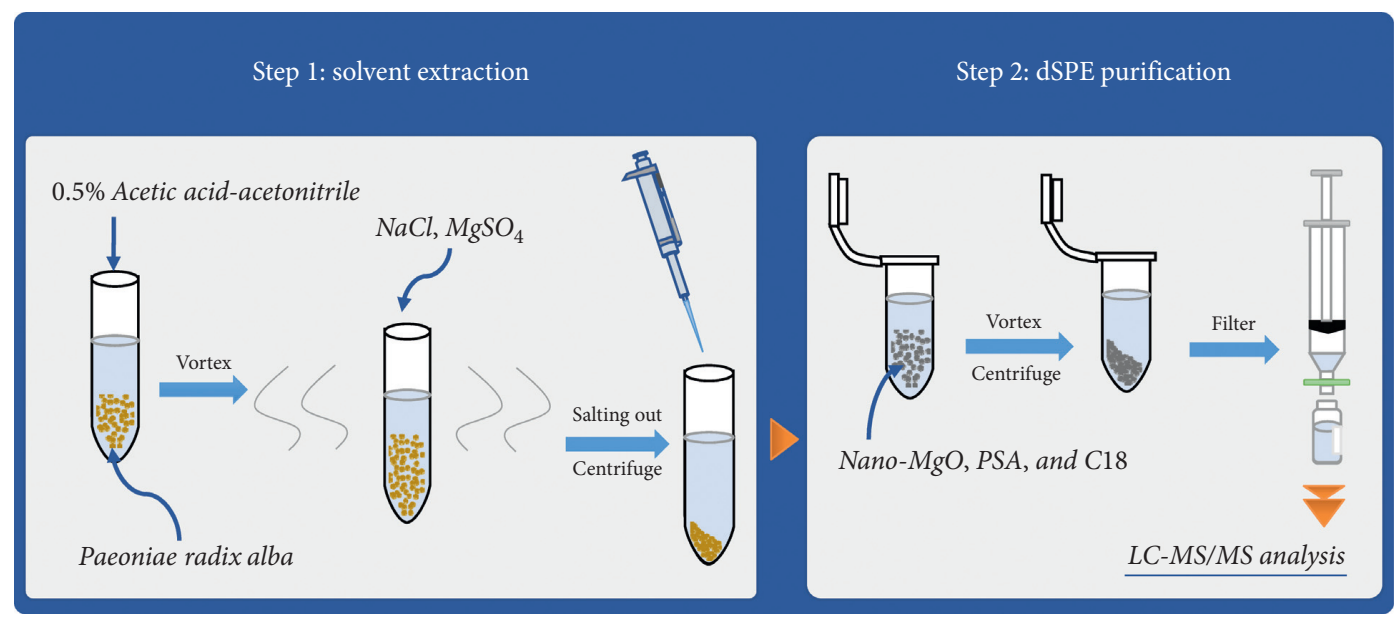

Figure 1: The scheme of the established pretreatment method procedure.

nano-MgO, PSA, and C18, were successively optimized. The extraction solution $(1 \mathrm{~mL})$ of the prespiked sample was transferred to a centrifuge tube containing different amounts of nano- $\mathrm{MgO}$ to explore the best purification effect. The amounts of PSA and C18 were sequentially tested based on the presence of nano-MgO. The recovery and matrix effect were used as the main parameters to determine the type and volume of extraction solvent and the optimal cleanup adsorbent dosage.

2.4. LC-MS/MS Analysis. The detection of 123 pesticides in the PRA samples via LC-MS/MS was carried out on a Nexera X2 LC system (Shimadzu, Kyoto, Japan) and Shimadzu LCMS-8050 with an electrospray ion source (ESI). An analytical column, ACE Excel 2 C18 $(100 \mathrm{~mm} \times 2.1 \mathrm{~mm}, 1.7 \mu \mathrm{m}$; Phenomenex, United Kingdom), was used for the chromatographic separation with a column temperature of $35^{\circ} \mathrm{C}$. The mobile phase consisted of $5 \mathrm{mmol} / \mathrm{L}$ ammonium formate aqueous solution (phase A) and methanol (phase B) with a gradient elution procedure. The gradient elution program is shown in Table $\mathrm{S} 1$. The total run time was $10 \mathrm{~min}$ and the flow rate was set at $0.3 \mathrm{~mL} / \mathrm{min}$, while the injection volume was $2 \mu \mathrm{L}$. The multiple reaction monitoring (MRM) mode was adopted for tandem spectrometry. The optimal collision energy and MRM precursor ion, product ion, and declustering potential for all target analytes are shown in Table S2. The retention time of each pesticide was $2 \mathrm{~ms}$. The electrospray ionization source was used with positive and negative ion modes. The temperature of the ESI capillary, heart block, and $\mathrm{DL}$ was $300^{\circ} \mathrm{C}, 400^{\circ} \mathrm{C}$, and $250^{\circ} \mathrm{C}$, respectively. Both the heating and drying gas flow were set at $10 \mathrm{~L} / \mathrm{min}$, while the flow rate of the nebulizing gas was set at $3 \mathrm{~L} / \mathrm{min}$.

2.5. Method Validation. The calibration curves of the analytes were fabricated by plotting the peak area as a function of the concentration and were used to evaluate the linearity of the pesticides. Both matrix-matched and solvent standard solutions were prepared with the concentration of each analyte at $0.5,1,2,5,10,20,50,100$, 200 , and $250 \mu \mathrm{g} / \mathrm{L}$. The recovery experiments were implemented by spiking five PRA samples with 123 pesticides at three different concentration levels $(20,50$, and $200 \mu \mathrm{g} / \mathrm{kg}$ ). The accuracy was evaluated with $3 \mathrm{rep}-$ licates for each spiked concentration level.

\section{Results and Discussion}

The recovery parameter was used to evaluate the extraction and purification efficiency, which is the ratio of the detected concentration to the spiked concentration [22]. The distribution of pesticide recovery was statistically divided and evaluated due to the presence of numerous pesticides. In the multi-pesticide residue analysis, a recovery range of $70-130 \%$ was required for accurate analysis, while $90-110 \%$ was more satisfactory.

3.1. Selection and Volume of the Extraction Solvent. The extraction solvent was selected to ensure efficient extraction of the target analyte and reduction of the interference of the coextracts. Three types of extraction solvents were compared, including pure acetonitrile, acetonitrile containing $0.5 \%$ acetic acid, and acetonitrile containing $1 \%$ acetic acid. The volume of extraction solvents was $8 \mathrm{~mL}$. The recovery distribution of the pesticides extracted by different solvents is shown in Figure 2(a). When acetonitrile was used as the extraction solvent, pesticide recovery was higher than $110 \%$, with $93.5 \%$ of pesticides with rates higher than $130 \%$. When acetic acid was added to acetonitrile, the rate of the analytes in the matrix was distinctly improved. When acetonitrile containing $0.5 \%$ acetic acid was used, $90.0 \%$ of the pesticides were recovered in the range from $75.7 \%$ to $128 \%$, with $48.0 \%$ of the pesticides with recoveries of 90-110\%. When the content of acetic acid in acetonitrile was increased to $1 \%, 77.2 \%$ of the pesticides were recovered with a range between $70 \%$ and $130 \%$, and only $17.1 \%$ of the pesticides were in the recovery range of 

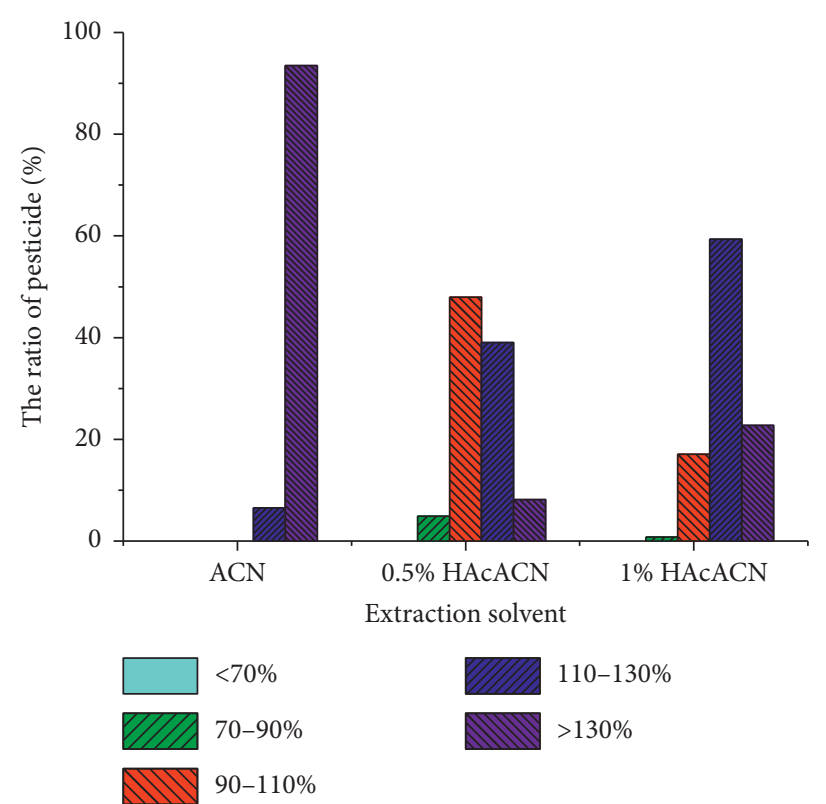

(a)

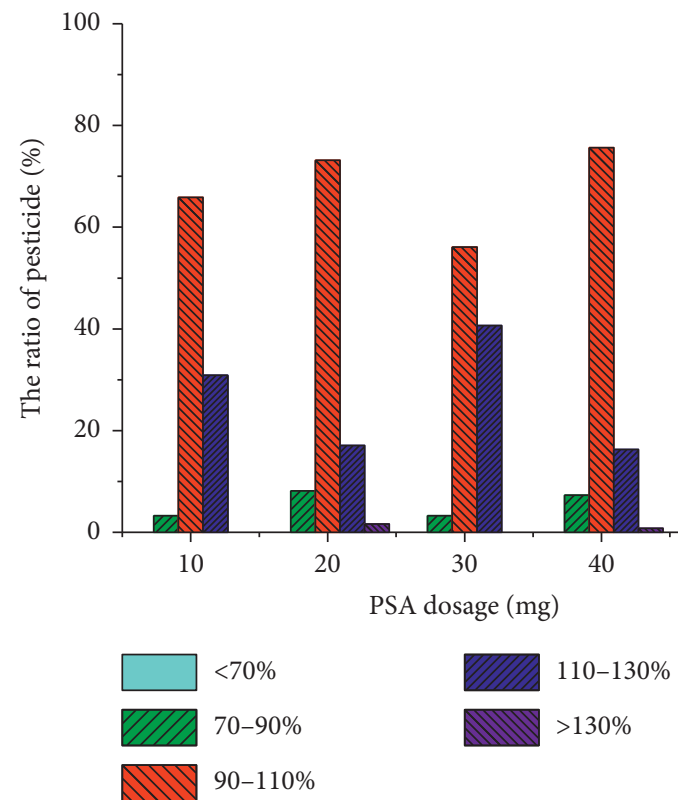

(c)

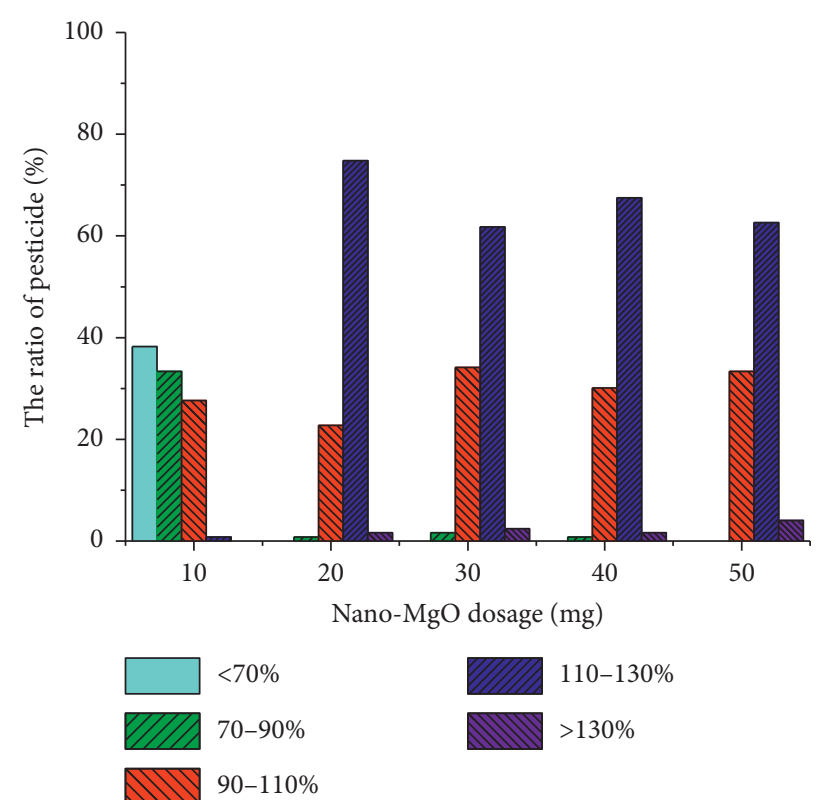

(b)

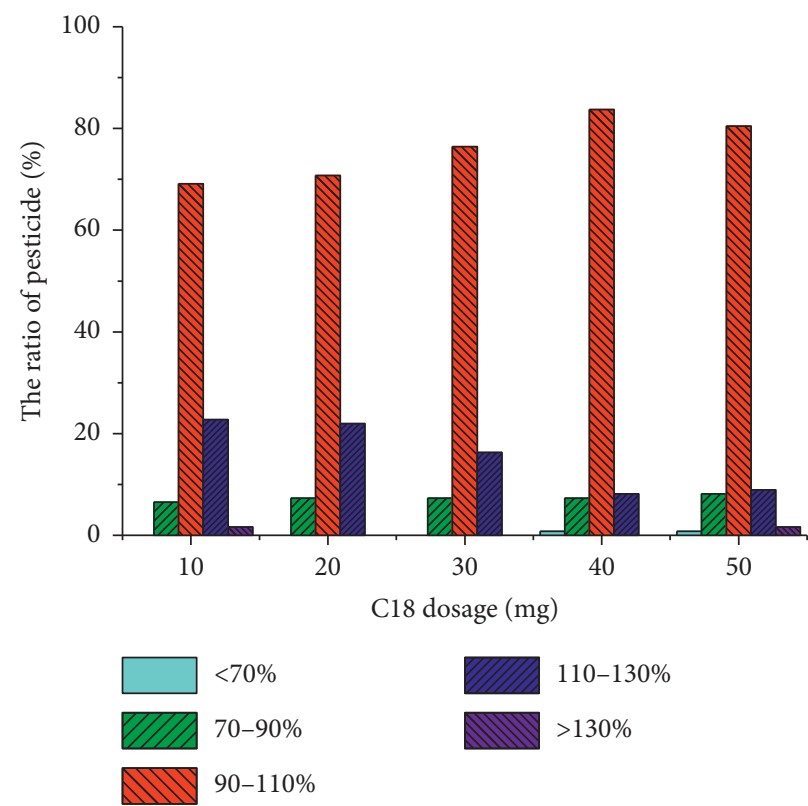

(d)

Figure 2: Influence of the extraction solvent (a), nano-MgO dosage (b), PSA dosage (c), and C18 dosage (d) on the pesticide recovery distribution.

90-110\%. Hence, acetonitrile containing $0.5 \%$ acetic acid was used as the extraction solvent.

After the extraction solvent was determined, the solvent volume was optimized. The spiked PRA samples were extracted with 6,8 , and $10 \mathrm{~mL}$ of acetonitrile containing $0.5 \%$ acetic acid. The recovery distribution of these pesticides was then calculated. The results (Figure S1) showed that when different volumes of extraction solvent were used, $71.5 \%(6 \mathrm{~mL}), 78.1 \%(8 \mathrm{~mL})$, and $76,4 \%$ $(10 \mathrm{~mL})$ of pesticides were in the recovery range of $90-110 \%$, respectively. It has been illustrated that $8 \mathrm{~mL}$ extraction solvent was better than $6 \mathrm{~mL}$ and $10 \mathrm{~mL}$. Hence, $8 \mathrm{~mL}$ acetonitrile containing $0.5 \%$ acetic acid was used in the further experiment.

\subsection{Optimization of the Purification Materials. Nano-MgO} was expected as a purification material to remove these acid compounds, such as benzoic acid and volatile oils in the PRA matrix. The nano-MgO dosage (10, 20, 30, 40, and $50 \mathrm{mg}$ ) was firstly optimized as the abovementioned method in section 2.3. The recoveries obtained with different amounts 
of nano- $\mathrm{MgO}$ are shown in Figure 2(b). It can be seen that when the dosage was $10 \mathrm{mg}$, the recovery of all pesticides was less than $110 \%$, with $38.2 \%$ of pesticides presenting recovery rates lower than $70 \%$. Recovery increased with increasing $\mathrm{MgO}$ content, specifically with more than $95.9 \%$ of pesticides observed in the recovery range of $70-130 \%$. When the amount of nano-MgO increased to $30 \mathrm{mg}, 34.2 \%$ of pesticides were in the recovery range of $90-110 \%$, which was maintained with increasing nano-MgO content. Under the premise of comprehensive consideration of recovery efficiency and $\mathrm{MgO}$ dosage, $30 \mathrm{mg}$ was chosen as the optimum nano-MgO content.

PSA is further employed as coadsorbent due to its characteristics as a weak anion exchanger, which can be used to remove oil, fatty acids, and waxy interferences. The combination of PSA and nano-MgO was expected to effectively remove a wide variety of impurities. The various amounts of PSA $(10,20,30,40$, and $50 \mathrm{mg})$ were studied with the presence of $30 \mathrm{mg}$ nano-MgO. The experimental operation was performed according to the procedures described previously and the recovery rate distribution results are shown in Figure 2(c). When the dosage of PSA was $40 \mathrm{mg}$, $75.6 \%$ of pesticides were in the recovery range of $90-110 \%$, which was slightly higher than the other four dosages. Compared with the purification without PSA, the percentage of pesticides in the recovery range of $90-110 \%$ increased from $34.2 \%$ to $75.6 \%$. This fully demonstrates that additional components were removed by adding PSA, and consequently, the recovery rates were improved.

C18 is reported to be useful in removing lipids and nonpolar interferences in the solid-phase extraction, so the component was added to the purification process. The amounts of C18 were set as 10, 20, 30, 40, and $50 \mathrm{mg}$, respectively. It can be clearly seen from Figure 2(d) that the pesticide recovery rate distribution was significantly better when $40 \mathrm{mg}$ of $\mathrm{C} 18$ was used. The proportion of pesticides with recovery rates in the range of $90-110 \%$ was as high as $83.7 \%$. Compared with the purification with nano-MgO and PSA alone, the proportion of pesticides in the range of $90-110 \%$ increased by $8.10 \%$. Hence, the ultimate purification materials in this method were nano-MgO $(30 \mathrm{mg})$, PSA (40 mg), and C18 (40 mg).

3.3. Purification Effect Evaluation. The matrix effect has been an inevitable problem in pesticide residue analysis based on LC-MS/MS [23], making quantitative analysis inaccurate. Hence, the matrix effect was used to evaluate the purification effect in the developed method. Typically, the evaluation of the matrix effect can be performed through two different methods. The first one is based on the peak area ratio $\left(R_{\mathrm{a}}\right)$ of the matrix-matched standard solution to the solvent standard solution at a certain concentration. The second one is based on the ratio of the slope of the matrix-matched standard solution curve to that of the solvent standard solution curve. The results obtained by the two different methods usually are in good agreement. Here, the peak area ratio was used in the optimization process. In general, a result in which $R_{\mathrm{a}}$ is equal to 1 means no matrix effect, and an $R_{\mathrm{a}}$ value within $0.8-1.2$ is considered an acceptable weak matrix effect $[24,25]$. The $R_{\mathrm{a}}$ values were divided into five groups to evaluate the matrix effect: $0-0.2,0.2-0.5,0.5-0.8$, $0.8-1.2$, and $>1.2$. $R_{\mathrm{a}}$ values within $0.8-1.2$ represented a weak matrix effect as mentioned before, and it is acceptable for an accurate analysis, while $R_{\mathrm{a}}$ values lower than 0.8 displayed signal suppression. Specifically, the smaller the $R_{\mathrm{a}}$ value, the stronger the signal suppression. Moreover, $R_{\mathrm{a}}$ values greater than 1.2 displayed signal enhancement.

The distribution of $R_{\mathrm{a}}$ values obtained from the results is displayed in Figure 3. Comparing the results without purification materials (Figure 3(a)), the percentage of pesticides with weak matrix effects at the optimum dosage of $30 \mathrm{mg}$ nano- $\mathrm{MgO}$ increased from $37.4 \%$ to $48.0 \%$. The results indicate that the addition of nano- $\mathrm{MgO}$ reduced the matrix effect of some of the pesticides. When PSA was added, $59.4 \%$ of pesticides exhibited $R_{\mathrm{a}}$ values within $0.8-1.2$, demonstrating the matrix effect of some of the pesticides had been further improved. With the addition of C18, more than $70.0 \%$ of pesticides showed a weak matrix effect. Additionally, the results in Figure 3(a) clearly demonstrate that the ratio of analytes with $R_{\mathrm{a}}$ values of $0.5-0.8$ decreased with the gradual addition of purification materials, whereas $R_{\mathrm{a}}$ values of $0.8-1.2$ increased. Moreover, it can be clearly seen from Figure 3(b) that for 15 pesticides, such as chlorantraniliprole, azoxystrobin, and azaconazole, the $R_{\mathrm{a}}$ values finally displayed weak or no matrix effect compared with the initial strong signal suppression and increasingly approached 1 in the optimization process. Most pesticides with a reduced matrix effect were triazole and amides. In particular, azaconazole, paclobutrazol, and myclobutanil showed the most significant improvement, with $R_{\mathrm{a}}$ values increased from $0.48,0.64$, and 0.62 to $0.90,0.98$, and 0.99 , respectively. In addition, the signal intensity before and after purification was compared, and the XIC chromatograms of four representative analytes are provided in Figure 4. Higher peak-heights of phosphamidon, atrazine, prochloraz, and fludioxonil were obtained after purification. The above results fully illustrate the superiority of the established method in improving matrix effects.

3.4. Method Validation. The feasibility and adaptability of the method were validated by evaluating linearity, sensitivity, matrix effect, and precision of the pesticide residue analysis in five PRA samples: PRA-ZJ, PRA-AH, PRA-SD, PRA-HN, and PRA-HB. The linear regression equations for five different PRA samples are provided in Tables S3-S7. In sample PRA-ZJ, $83.7 \%$ of the analytes were linear in the range of $0.5-250 \mu \mathrm{g} / \mathrm{L}$, and the correlation coefficient $(R)$ was higher than 0.99 . Because of the high detection sensitivity of the instrument for some of the compounds, the upper limit of the linear range could be reduced to $50 \mu \mathrm{g} / \mathrm{L}$ or $100 \mu \mathrm{g} / \mathrm{L}$ for some pesticides. Additionally, 14 pesticides were within the linear range from 0.5 to $100 \mu \mathrm{g} / \mathrm{L}$, including malaoxon, hexazinone, phorate sulfone, azoxystrobin, and flutolanil (Tables S3-S7). The linearity range of four other pesticides (vitavax, ethiofencarb, mepronil, and phosalone) was from 0.5 to $50 \mu \mathrm{g} / \mathrm{L}$. Moreover, the linear range of pesticides 


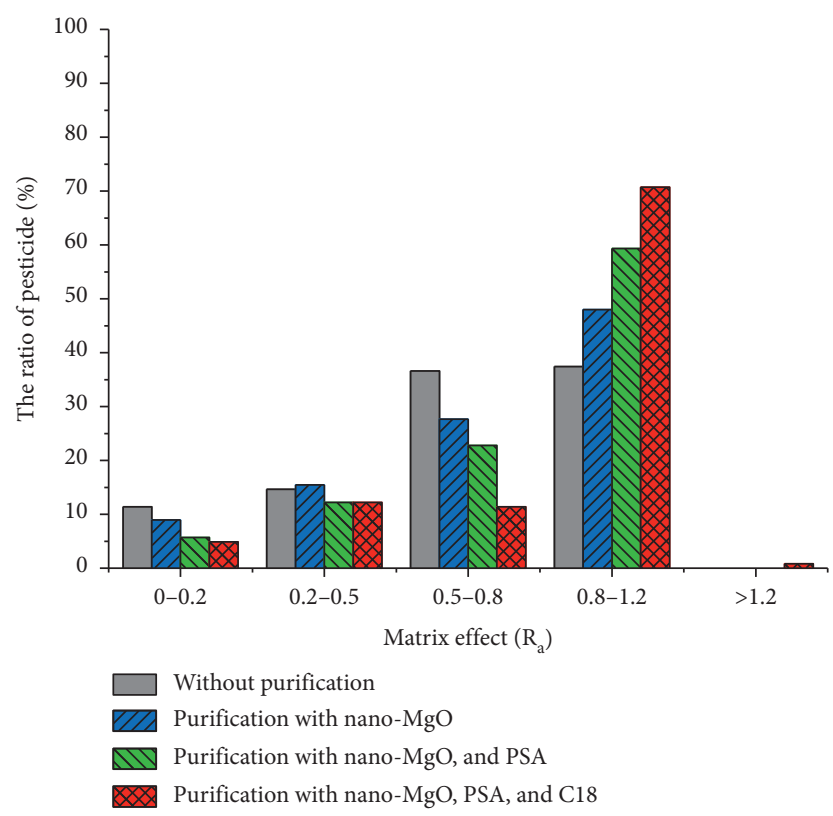

(a)

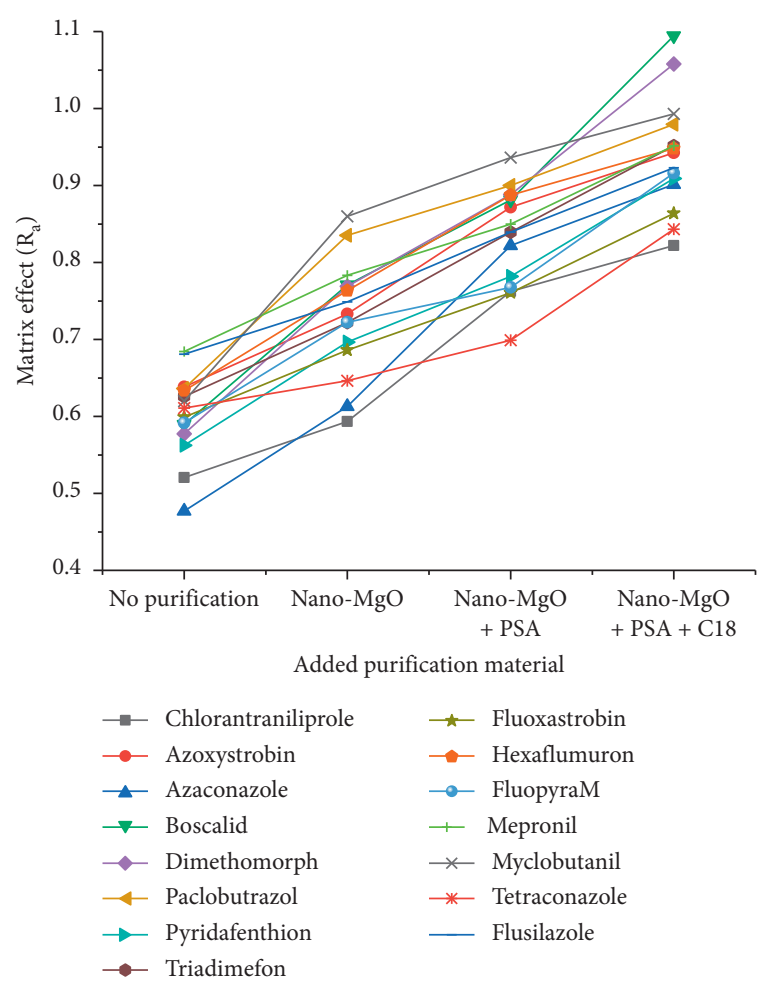

(b)

FIgURE 3: Effects of different purifications on matrix effect of pesticides (a) and fifteen representative pesticides (b).

changed slightly with different matrices. The limit of detection (LOD) was determined as three times the concentration of the signal to noise ratio of the instrument. As shown in Tables S3-S7, LODs were in the range of $0.16-4.91 \mu \mathrm{g} / \mathrm{kg}$ for sample PRA-ZJ, $0.14-4.99 \mu \mathrm{g} / \mathrm{kg}$ for samples PRA-AH and PRA-SD, $0.11-4.98 \mu \mathrm{g} / \mathrm{kg}$ for PRA$\mathrm{HN}$, and $0.16-4.98 \mu \mathrm{g} / \mathrm{kg}$ for PRA-HB.

The limit of quantification (LOQ) of the method was determined according to the European Commission guidance document SANTE/12682/2019 [26]. In the present work, the lowest spiked concentration of the pesticides was $20 \mu \mathrm{g} / \mathrm{kg}$. Satisfactory recoveries were achieved for $94.0 \%$ of the pesticides at this concentration, and thus the method LOQ of these pesticides was $20 \mu \mathrm{g} / \mathrm{kg}$. Other six pesticides, such as 3-hydroxy-carbofuran, abamectin, clothianidin, dicrotophos, monocrotophos, and terbufos, showed satisfactory recoveries at $50 \mu \mathrm{g} / \mathrm{kg}$. Therefore, the method LOQ of these six pesticides was determined to be $50 \mu \mathrm{g} / \mathrm{kg}$.

The matrix effect was evaluated by comparing the ratio of the slope of the matrix to the solvent-matched curves in the validation process [27]. The results of the matrix effect are shown in Tables S3-S7. The pesticides with a ratio from 0.8 to 1.2 for PRA-ZJ, PRA -AH, PRA-SD, PRA-HN, and PRA$\mathrm{HB}$ account for $61.8 \%, 58.5 \%, 54.5 \%, 63.4 \%$, and $59.4 \%$ of all pesticides, respectively. This shows that the method was applicable to five different PRA samples, and more than half of the analytes had acceptable matrix effects.

In addition, recovery experiments were used to evaluate the accuracy and precision of the method. The detailed results of the recovery and the relative standard deviation
(RSD) for all pesticides in five different PRA samples at three concentration levels are shown in Tables S8-S12. The mean of the recovery rate and RSDs and the number of pesticides not detected at each spiked concentration are collated in Table 1. It was determined that the mean of the recovery rates of the five different PRA samples ranged from $98 \%$ to $111 \%$ and the mean RSD was less than $6.00 \%$. The overall average recovery between all the PRA samples for the concentrations of 20,50 , and $200 \mu \mathrm{g} / \mathrm{kg}$ was $103 \%, 106 \%$, and $103 \%$, respectively, with mean RSD values of $4.83 \%, 3.82 \%$, and $2.87 \%$. When the spiked concentration was $20 \mu \mathrm{g} / \mathrm{kg}$, the number of undetected analytes ranged from 6 to 17 . When the concentration increased to $200 \mu \mathrm{g} / \mathrm{kg}$, all pesticides were detected. More than $78.9 \%$ of the detected pesticides had satisfactory recovery rates on the three concentration levels in the five PRA samples. The number of pesticides with recovery rates between $70 \%$ and $120 \%$ at each concentration level in each PRA sample is shown in Table 1. Based on the aforementioned results, it can be confirmed that the method has satisfactory precision.

3.5. Method Comparison. The performance of this method was compared with that of the traditional QuEChERS method [8], in which no acid is added in the extraction process and the extraction solution is purified by $\mathrm{C} 18$ and PSA. Sample PRA-ZJ, at a spiking concentration of $0.2 \mathrm{mg} /$ $\mathrm{kg}$, was extracted and purified by the two methods, and the results of the recovery rates and matrix effect are shown in Figure 5 . The recovery rate and $R_{\mathrm{a}}$ value distribution were 

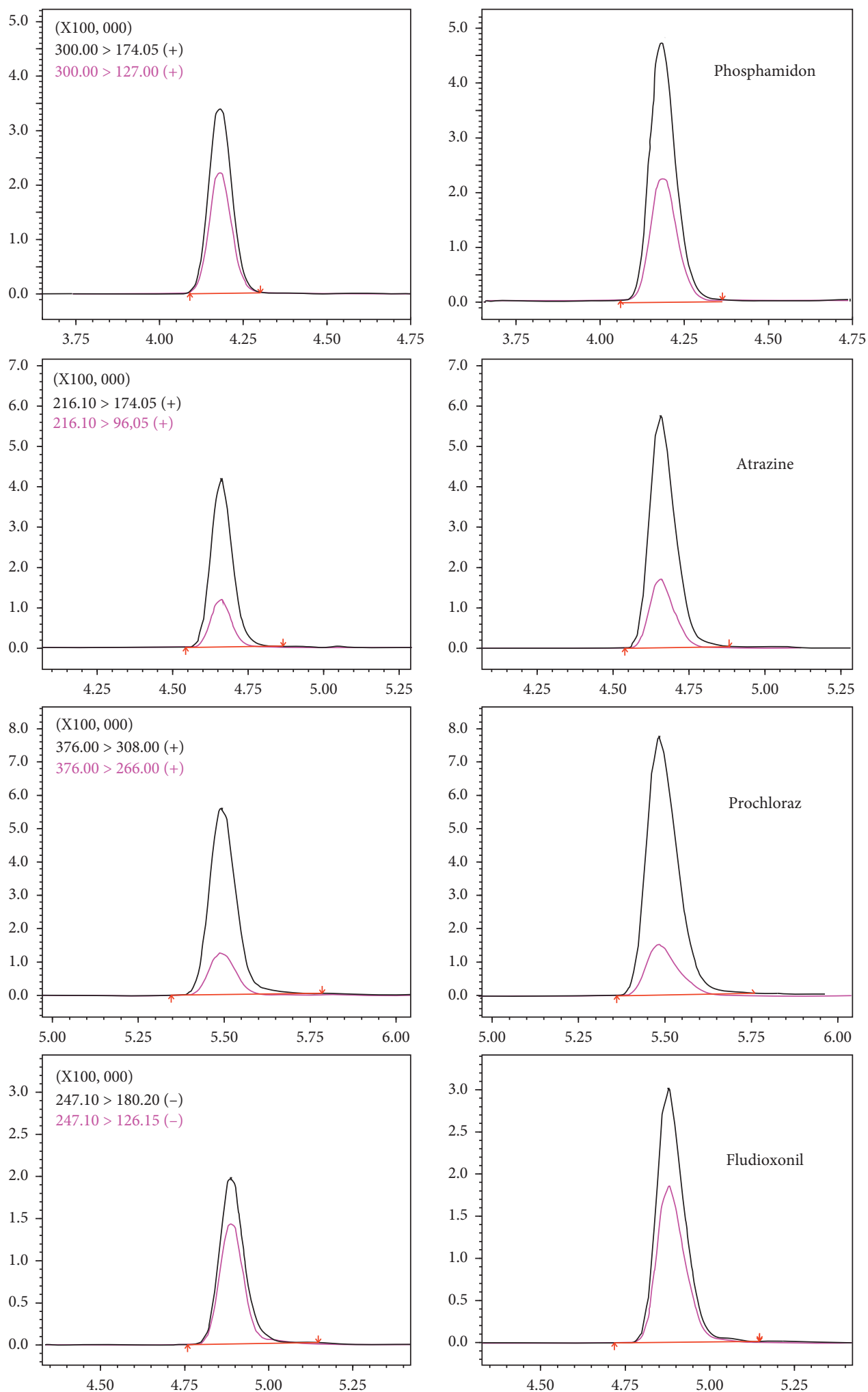

(a)

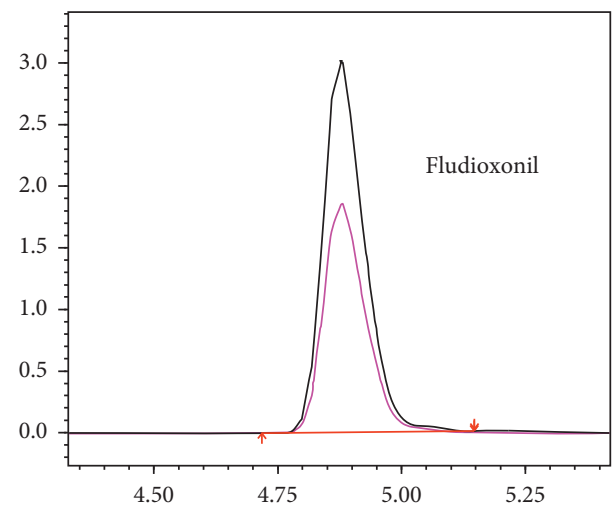

(b)

FigURE 4: XIC chromatograms of four representative pesticides at $40 \mu \mathrm{g} / \mathrm{L}$ in unpurified solution (a) and purified solution (b).

compared to analyze the two methods. As shown in Figure 5(a), compared with the traditional QuEChERS method, the percentage of pesticides with recovery rates in the range of $90-110 \%$ increased from $7.32 \%$ to $83.7 \%$, demonstrating a significant improvement in the recovery of pesticides by using the present method. In terms of the 
TABLE 1: Recoveries of the spiked pesticides from the various PRA samples at pesticide concentrations of 20,50 , and $200 \mu \mathrm{g} / \mathrm{kg}$ and the number of pesticides not detected (ND) at each concentration.

Recoveries \pm RSD (\%) for pesticides and the number of nondetects at 20, 50, and $200 \mu \mathrm{g} / \mathrm{kg}$ in
each PRA

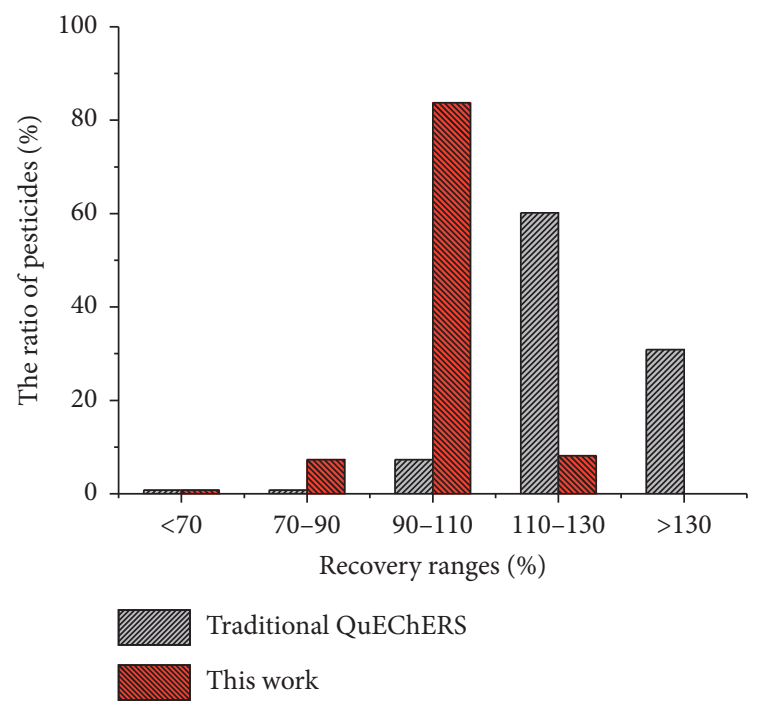

(a)

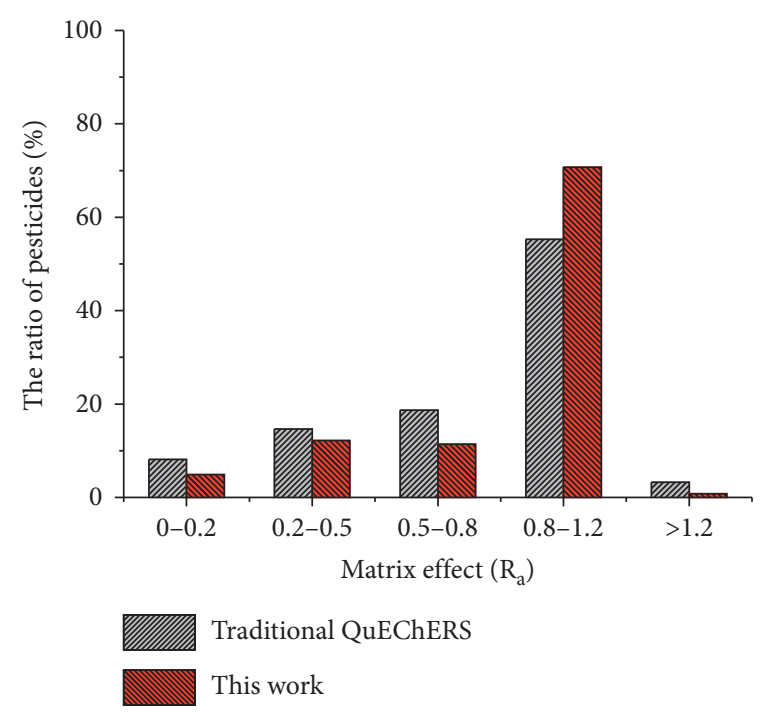

(b)

FIGURE 5: Comparison of recovery (a) and matrix effect (b) of the developed method with the traditional QuEChERS method for pesticides analysis of PRA-ZJ sample.

TABle 2: Comparison of method performance with the reported method for analysis of Paeoniae Radix Alba.

\begin{tabular}{|c|c|c|c|c|c|c|}
\hline Methods & Analytes & $\begin{array}{l}\text { Amounts of } \\
\text { samples (g) }\end{array}$ & $\begin{array}{l}\text { Volume of organics } \\
(\mathrm{mL})\end{array}$ & Purification materials & $\begin{array}{c}\text { Mean recovery } \\
(\%)\end{array}$ & References \\
\hline $\begin{array}{l}\text { SPE-GC-MS/ } \\
\text { MS }\end{array}$ & 99 pesticides & 4 & 20 & SPE column & $66.7-128$ & {$[11]$} \\
\hline $\begin{array}{l}\text { dSPE-HPLC- } \\
\text { MS }\end{array}$ & 23 pesticides & 5 & 20 & PSA & $70.0-120$ & {$[13]$} \\
\hline SPE-UFLC & $\begin{array}{l}5 \text { triazine } \\
\text { herbicides }\end{array}$ & 1 & 5 & $\begin{array}{l}\text { Molecularly imprinted } \\
\text { polymers }\end{array}$ & $92.4-107$ & {$[28]$} \\
\hline SPE-GC-ECD & 20 pesticides & 5 & 40 & Silica gel column & $74.4-115$ & [29] \\
\hline $\begin{array}{l}\text { dSPE-LC-MS/ } \\
\text { MS }\end{array}$ & 123 pesticides & 2 & 8 & Nano-MgO, PSA, C18 & $98.0-111$ & This work \\
\hline
\end{tabular}

matrix effect (Figure 5(b)), the proportion of pesticides with an $R_{\mathrm{a}}$ value in the range of $0.8-1.2$ increased from $55.3 \%$ to $70.7 \%$. It can be hypothesized that the matrix effect of the analytes was improved due to the introduction of nano$\mathrm{MgO}$. As a summary of the above results, the improved method displayed a better ability to solve the complexity of PRA matrices.
The performance of the established method for PRA sample analysis was compared with other previously reported methods (Table 2). The present study was rapid, simple, and convenient due to the improvement of the traditional QuEChERS method. The advantages of the present method include low sample and organic solvent consumption, cheap purification material, and no special 
instrument required during the operation $[11,13,28,29]$. The established method can simultaneously determine 123 pesticides in five different PRA samples to achieve highthroughput analysis and wide applicability. In addition, this method has outstanding advantages in reducing the matrix effect.

\section{Conclusions}

A rapid and efficient multiresidue analytical method was developed to detect 123 pesticides residues in various PRA samples with nano- $\mathrm{MgO}$ as dispersive solid-phase adsorbent. The cleanup adsorbent combination of nano- $\mathrm{MgO}$, $\mathrm{C} 18$, and PSA was proved to be excellent in reducing the matrix effect, providing accurate quantitative results of the analytes. A systematic method validation for five different PRA samples was performed to evaluate linearity, LODs, matrix effect, recoveries, and RSDs, demonstrating the satisfactory sensitivity, precision, accuracy, and universality of the method. Furthermore, the developed method was effective, and with a high throughput when compared with other reported methods. Nano-MgO was used as a cleanup material for the first time to analyze pesticides residues in PRA samples, demonstrating an efficient purification performance and good application prospects in a complex matrix. The established method could fully meet the multiple pesticide residue analysis requirements for PRA samples.

$\begin{array}{ll}\text { Abbreviations } \\ \text { C18: } & \text { Octadecylsilane } \\ \text { dSPE: } & \text { Dispersive solid-phase extraction } \\ \text { HAc: } & \text { Acetic acid } \\ \text { HPLC: } & \text { High-performance liquid chromatography } \\ \text { LC-MS/ } & \text { Liquid chromatography-tandem mass } \\ \text { MS: } & \text { spectrometry } \\ \text { MRM: } & \text { Multiple reaction monitoring } \\ \text { MgSO } & \text { Anhydrous magnesium sulfate } \\ \text { NaCl: } & \text { Sodium chloride } \\ \text { PSA: } & \text { Primary secondary amine } \\ \text { PRA: } & \text { Paeoniae Radix Alba } \\ \text { RSD: } & \text { Relative standard deviation. }\end{array}$

\section{Data Availability}

The data used to support the findings of this study are included within the supplementary information files owing to the equal contribution

\section{Disclosure}

Xinquan Wang is the co-first author.

\section{Conflicts of Interest}

The authors have declared no conflicts of interest.

\section{Authors' Contributions}

Xinquan Wang contributed to this work.

\section{Acknowledgments}

This work was supported by the Key Research and Development Program of Zhejiang Province (Grant no. 2020C02023) and the fund of State Key Laboratory for Quality and Safety of Agro-Products (Grant nos. 2010DS700124-KF1905 and 2010DS700124-ZZ2009).

\section{Supplementary Materials}

The influence of the volume of the extraction solvent is shown in Figure S1. The gradient elution program is shown in Table S1. The optimal collision energy and MRM precursor ion, product ion, and declustering potential for all target analytes are shown in Table S2. The results of the matrix effect are shown in Tables S3-S7. The detailed results of the recovery rates and the relative standard deviation (RSD) for all pesticides in five different PRA samples at three concentration levels are shown in Tables S8-S12. (Supplementary Materials)

\section{References}

[1] Y. Fu, X. Dou, L. Zhang, J. Qin, M. Yang, J. Luo, A comprehensive analysis of 201 pesticides for different herbal species-ready application using gas chromatography-tandem mass spectrometry coupled with QuEChERs," Journal of Chromatography B, vol. 1125, 2019.

[2] J. Xiao, X. Xu, F. Wang et al., "Analysis of exposure to pesticide residues from Traditional Chinese Medicine," Journal of Hazardous Materials, vol. 365, pp. 857-867, 2019.

[3] Z. Zhang, T. Bo, Y. Bai et al., "Quadrupole time-of-flight mass spectrometry as a powerful tool for demystifying traditional Chinese medicine," TrAC Trends in Analytical Chemistry, vol. 72, pp. 169-180, 2015.

[4] L. Yang, H. Jiang, X. Y. Guo et al., "Quantitative analysis of different batches of raw, wineprocessed, and vinegar-processed Paeoniae Alba Radix using ultra-performance convergence chromatography coupled with photo diode array detection," Biomedical Chromatography, vol. 33, Article ID e4485, 2019.

[5] Z. K. Qiu, J. L. He, X. Liu, J. Zeng, J. S. Chen, and H. Nie, "Anti-PTSD-like effects of albiflorin extracted from radix paeoniae alba," Journal of Ethnopharmacology, vol. 198, pp. 324-330, 2017.

[6] Y. Wang, S. M. Gao, R. Li, M. Zhang, S. Gao, and C. Q. Yu, "Antidepressant-like effects of the radix bupleuri and radix paeoniae Alba drug pair," Neuroscience Letters, vol. 633, pp. 14-20, 2016.

[7] X. Li, M. Wang, J. Zhao et al., "Ultrasound-assisted emulsification liquid phase microextraction method based on deep eutectic solvent as extraction solvent for determination of five pesticides in traditional Chinese medicine," Journal of Pharmaceutical and Biomedical Analysis, vol. 166, pp. 213221, 2019.

[8] J. G. Wang, Y. X. Wei, F. Wang, X. F. Liu, Y. C. Wang, and H. Guo, "Rapid determination of 85 pesticide residues in Chinese medicinal herbs by triple quadrupole gas chromatography-tandem mass spectrometry," Chinese Journal of Health Laboratory, vol. 27, pp. 324-330, 2017. 
[9] J. F. Wang, S. S. Zhou, P. Wang, X. Xue, and F. Fang, "Simultaneous determination of 10 pesticide residues of organophosphorus in radix paeoniae Alba by gas chromatography-tandem mass spectrometry method," Chemical Analysis and Meterage, vol. 22, pp. 49-52, 2013.

[10] R. Xu, J. Wu, Y. Liu et al., "Analysis of pesticide residues using the Quick Easy Cheap Effective Rugged and Safe (QuEChERS) pesticide multiresidue method in traditional Chinese medicine by gas chromatography with electron capture detection," Chemosphere, vol. 84, no. 7, pp. 908-912, 2011.

[11] X. Liu, L. Tong, W. Meng, and G. Sun, "Determination of 99 pesticide residues inPaeoniae Radix Albaby gas chromatography-triple quadrupole tandem mass spectrometry," Chinese Journal of Chromatography, vol. 33, no. 8, pp. 869-877, 2015.

[12] X. Shen, X. H. Chen, D. A. Guo, S. T. Zhang, and K. S. Bi, "Determination of 20 pesticide residues in Radix Paeoniae Alba by hollow fiber-based liquid phase microextraction coupled with gas chromatography," Chinese Journal of Analytical Chemistry, vol. 29, pp. 1745-1748, 2009.

[13] Y. Liu, J. R. Gong, X. H. Mao, S. Ji, and S. F. Wang, "Determination of 23 pesticide residues in Paeoniae radix Alba using high performance liquid chromatography coupled with mass spectrometry," Chinese Journal of Pesticide Science, vol. 13, pp. 496-502, 2011.

[14] B. Holmes, A. Dunkin, R. Schoen, and C. Wiseman, "Singlelaboratory ruggedness testing and validation of a modified QuEChERS approach to quantify 185 pesticide residues in salmon by liquid chromatography- and gas chromatographytandem mass spectrometry," Journal of Agricultural and Food Chemistry, vol. 63, no. 21, pp. 5100-5106, 2015.

[15] V. C. Fernandes, W. Luts, C. Delerue-Matos, and V. F. Domingues, "Improved QuEChERS for analysis of polybrominated diphenyl ethers and novel brominated flame retardants in capsicum cultivars using gas chromatography," Journal of Agricultural and Food Chemistry, vol. 68, no. 10, pp. 3260-3266, 2020.

[16] Z. Zhang, M. Dong, X. Hao, L. Han, S. Song, and W. Yao, "Evaluation of cleanup procedures in pesticide multi-residue analysis with QuEChERS in cinnamon bark," Food Chemistry, vol. 276, pp. 140-146, 2019.

[17] J. Liu, L. Tong, D. Li et al., "Comparison of two extraction methods for the determination of 135 pesticides in Corydalis Rhizoma, Chuanxiong Rhizoma and Angelicae Sinensis Radix by liquid chromatography-triple quadrupole-mass spectrometry. Application to the roots and rhizomes of Chinese herbal medicines," Journal of Chromatography B, vol. 10171018, pp. 233-240, 2016.

[18] J. Zhou, Y. Xia, Y. Gong, W. Li, and Z. Li, "Efficient natural organic matter removal from water using nano-MgO coupled with microfiltration membrane separation," Science of The Total Environment, vol. 711, Article ID 135120, 2020.

[19] Y. Xia, K. Dong, X. Xiang, W. Li, Y. Gong, and Z. Li, "Phosphorus hyperaccumulation in nano-MgO using a circular recovery process based on multiple phase transitions from periclase to brucite," Science of The Total Environment, vol. 727, Article ID 138510, 2020.

[20] P. Jeevanandam and K. J. Klabunde, "A study on adsorption of surfactant molecules on magnesium oxide nanocrystals prepared by an aerogel route," Langmuir, vol. 18, no. 13, pp. 5309-5313, 2002.

[21] J. W. Lu, W. Wang, A. Q. Zhang, X. L. Qiao, and J. G. Chen, "Research progress of the adsorption and decomposition properties of Nano-MgO," Bull Chin Ceram Soc, vol. 30, pp. 1094-1098, 2011.
[22] B. Zhu, X. Xu, J. Luo et al., "Simultaneous determination of 131 pesticides in tea by on-line GPC-GC-MS/MS using graphitized multi-walled carbon nanotubes as dispersive solid phase extraction sorbent," Food Chemistry, vol. 276, pp. 202-208, 2019.

[23] P. Parrilla Vázquez, C. Ferrer, M. J. Martínez Bueno, and A. R. Fernández-Alba, "Pesticide residues in spices and herbs: sample preparation methods and determination by chromatographic techniques," TrAC Trends in Analytical Chemistry, vol. 115, pp. 13-22, 2019.

[24] B. K. Matuszewski, M. L. Constanzer, and C. M. Chavez-Eng, "Strategies for the assessment of matrix effect in quantitative bioanalytical methods based on HPLC-MS/MS," Analytical Chemistry, vol. 75, no. 13, pp. 3019-3030, 2003.

[25] S. Wang, P. Qi, S. Di et al., "Significant role of supercritical fluid chromatography - mass spectrometry in improving the matrix effect and analytical efficiency during multi-pesticides residue analysis of complex chrysanthemum samples," Analytica Chimica Acta, vol. 1074, pp. 108-116, 2019.

[26] SANTE/12682/2019-Guidance Document on Analytical Quality Control and Method Validation Procedures for Pesticide Residues and Analysis in Food and Feed, (2019).

[27] F. Gosetti, E. Mazzucco, D. Zampieri, and M. C. Gennaro, "Signal suppression/enhancement in high-performance liquid chromatography tandem mass spectrometry," Journal of Chromatography A, vol. 1217, no. 25, pp. 3929-3937, 2010.

[28] X. Li, Y. Wang, Q. Sun, B. Xu, Y. Yu, and X. Wang, "Molecularly imprinted solid phase extraction in a syringe filter for determination of triazine herbicides in Radix Paeoniae Alba by ultra-fast liquid chromatography," Talanta, vol. 148, pp. 539-547, 2016.

[29] Q. Gao, R. M. Hua, F. Tang et al., "A multiresidue method for 20 pesticides in Radix paeoniae Alba of Chinese herb by gas chromatography with electron-capture detection," The Bulletin of Environmental Contamination and Toxicology, vol. 84, pp. 779-783, 2010. 